Supporting Information

\title{
Photothermal-responsive graphene oxide membrane with smart gates for water purification
}

Yang Wang, Zheng Zhang, Ting Li, Piming Ma, Hongji Zhang, Mingqing Chen, Mingliang Du, Weifu Dong*

Key Laboratory of Synthetic and Biological Colloids, Ministry of Education, School of Chemical and Material Engineering, Jiangnan University, 1800 Lihu Road, Wuxi 214122, China;

*E-mail: wfdong@jiangnan.edu.cn

Total pages: 10 (S1-S10)

\section{Experimental Section}

Preparation of UPy-NCO

Modification of PGO and PCNT with UPy-NCO

\section{Figures}

Figure S1. ${ }^{1} \mathrm{H}$ NMR spectrum of UPy-NCO.

Figure S2. ATR-FTIR spectra of GO, PGO, PGO-UPy, PCNTs and PCNT-UPy.

Figure S3. Full-scale XPS spectra of GO and PGO-UPy (a), N 1s of PGO-UPy.

Figure S4. Raman spectra of GO and PGO-UPy.

Figure S5. Raman spectra of MWCNT and PCNT-UPy.

Figure S6. TGA curves of GO, PGO, PGO-UPy, MWCNTs, PCNTs and PCNT-UPy.

Figure S7. Cross-section SEM images of PGO/PCNT-UPy membrane.

Figure S8. XRD of pure GO membrane (a), and PGO/PCNT-UPy before (b) and after (c) irradiation of NIR.

Figure S9. Water contact angle of pure GO and PGO/PCNT-UPy membrane.

Figure S10. Thickness-dependent water permeance and gating ratio of PGO/PCNT-UPy membranes.

Figure S11. 3D molecular schematic illustrations of methyl orange (MO), rhodamine B (RhB) and coomassie brilliant blue (CBB). 


\section{Experimental details for preparation of UPy-NCO}

In briefly, 2-amino-4-hydroxy-6-methylpyrimidine (5.0 g, $40 \mathrm{mmol}, 1 \mathrm{eq})$ and HDI (40.32g, $240 \mathrm{mmol}, 6 \mathrm{eq})$ were heated in a flask at $100^{\circ} \mathrm{C}$ for $20 \mathrm{~h}$ under a $\mathrm{N}_{2}$ atmosphere. Pentane was added to the flask for precipitation after the mixture was cooled down to the room temperature. The precipitate was filtered and washed with pentane followed by drying in vacuum at $40^{\circ} \mathrm{C}$ for $12 \mathrm{~h}$.

\section{Experimental details for modification of PGO and PCNT with UPy-NCO}

The PGO (or PCNT) and anhydrous $N, N$-dimethylformamide were charged into round bottom flask under a nitrogen atmosphere. The mixture was stirred and sonicated for $30 \mathrm{~min}$. After the complete dispersing of the PGO (or PCNT), a catalytic amount of dibutyltin dilaurate (DBTDL) (1 drop) and UPy-NCO were added and the mixture was then stirred at $100^{\circ} \mathrm{C}$ for $16 \mathrm{~h}$ under the protection of nitrogen. The reaction mixture was purified via filtration, the powder was thoroughly washed with DMF. After drying at $45^{\circ} \mathrm{C}$ in vacuum for overnight, UPy group functionlized PGO (or PCNT) were obtained. 
Synthesis of UPy-NCO. UPy-NCO was synthesized according to the procedure. 1H NMR (400 $\mathrm{MHz}, \mathrm{CDCl} 3): \delta=13.12\left(\mathrm{~s}, 1 \mathrm{H}, \mathrm{CH}_{3} \mathrm{CN} \underline{\mathrm{H}}\right), 11.87\left(\mathrm{~s}, 1 \mathrm{H}, \mathrm{CH}_{2} \mathrm{NH}(\mathrm{C}=\mathrm{O}) \mathrm{N} \underline{\mathrm{H}}\right), 10.19(\mathrm{~s}, 1 \mathrm{H}$, $\left.\mathrm{CH}_{2} \mathrm{~N} \underline{\mathrm{H}}(\mathrm{C}=\mathrm{O}) \mathrm{NH}\right), 5.83\left(\mathrm{~s}, 1 \mathrm{H}, \mathrm{CH}=\mathrm{CCH}_{3}\right), 3.27\left(\mathrm{~m}, 4 \mathrm{H}, \mathrm{NH}(\mathrm{C}=\mathrm{O}) \mathrm{NHC}_{2}+\underline{\mathrm{C}}_{2} \mathrm{NCO}\right), 2.24(\mathrm{~s}$, $\left.3 \mathrm{H}, \underline{\mathrm{CH}}_{3} \mathrm{C}=\mathrm{CH}\right), 1.62\left(\mathrm{~m}, 4 \mathrm{H}, \mathrm{NCH}_{2} \mathrm{C}_{2}\right), 1.41\left(\mathrm{~m}, 4 \mathrm{H}, \mathrm{CH}_{2} \mathrm{CH}_{2} \mathrm{C}_{2} \mathrm{C}_{2} \mathrm{CH}_{2} \mathrm{CH}_{2} \mathrm{NCO}\right.$ ) (as shown in Figure S1).

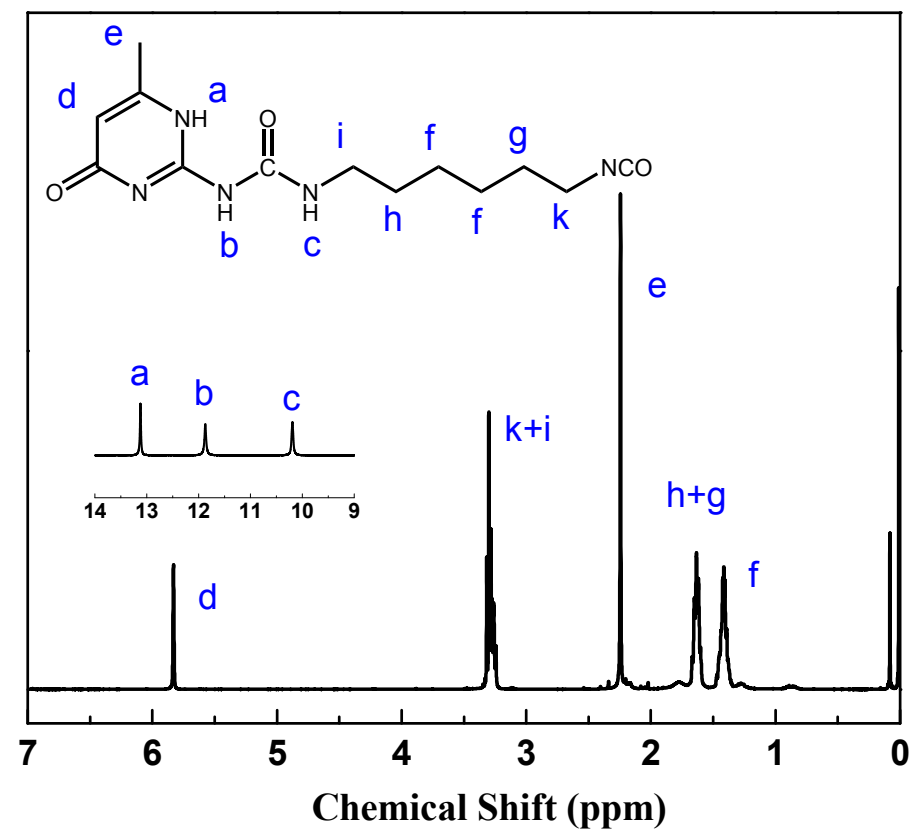

Figure S1. ${ }^{1} \mathrm{H}$ NMR spectrum of UPy-NCO. 


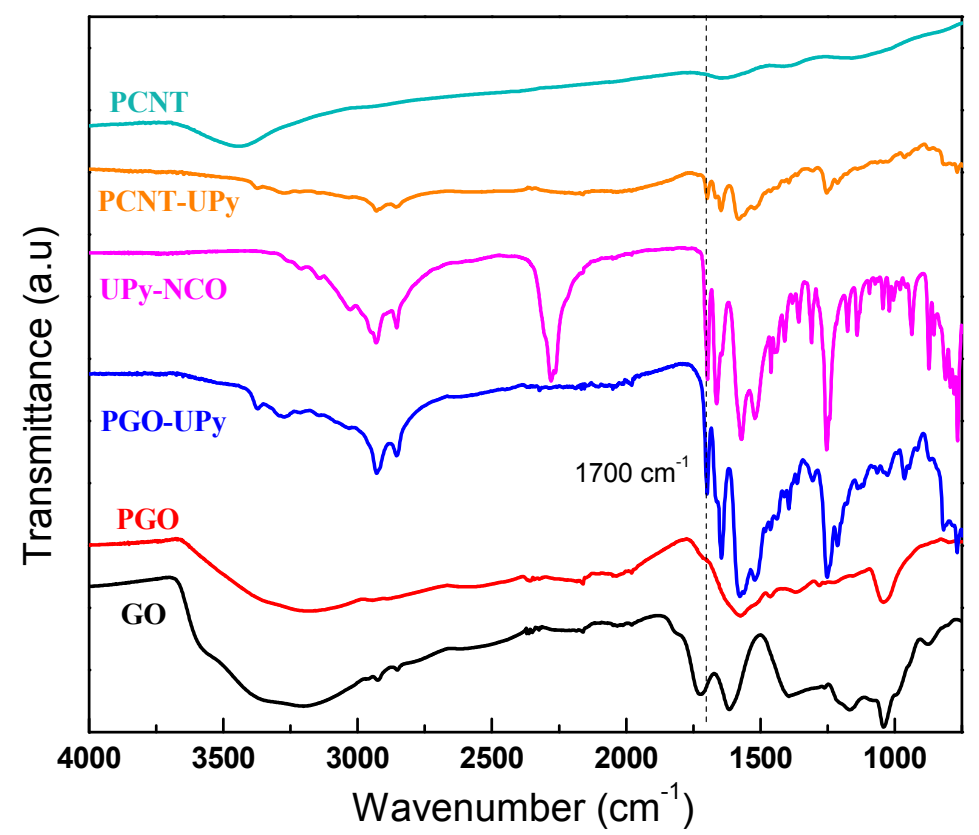

Figure S2. ATR-FTIR spectra of GO, PGO, PGO-UPy, PCNTs and PCNT-UPy.
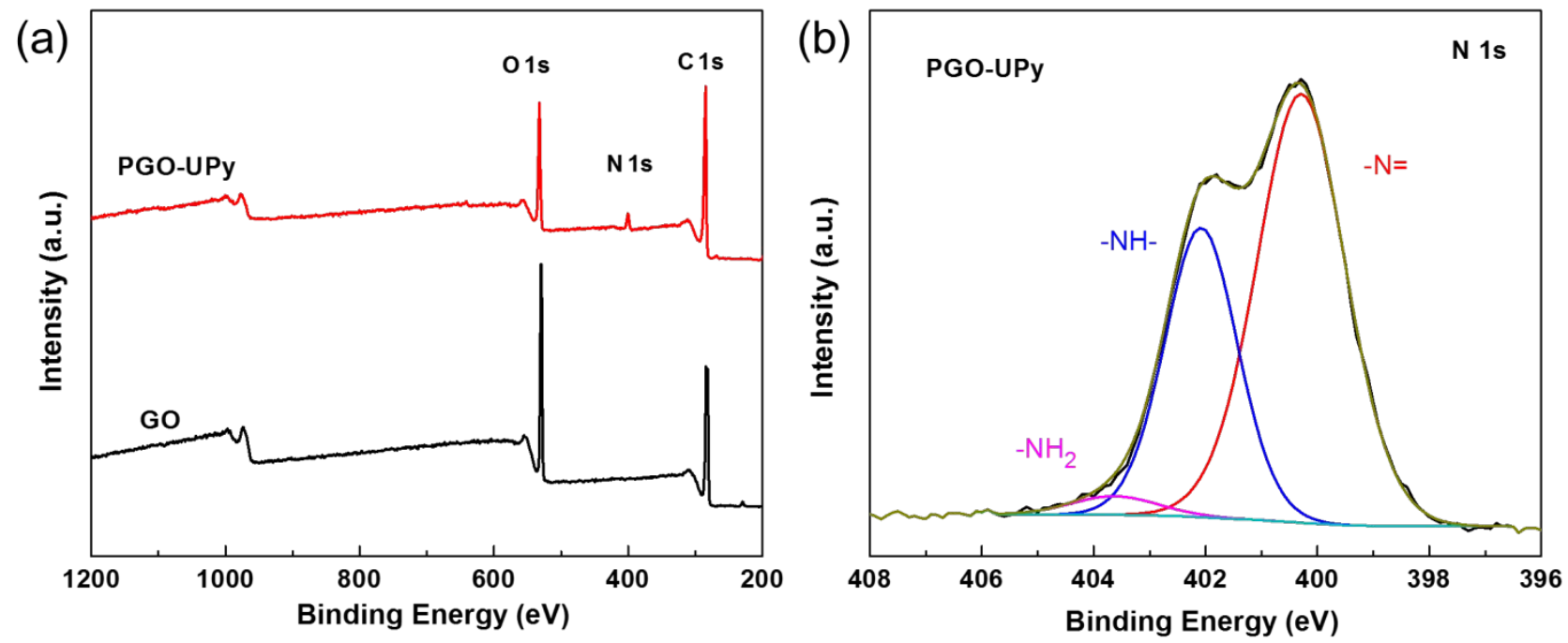

Figure S3. Full-scale XPS spectra of GO and PGO-UPy (a), N 1s of PGO-UPy (b).

Figure $\mathbf{S 3}$ shows the XPS spectra of GO and PGO-UPy. It is noticed that a new characteristic $\mathrm{N}$ 1s peak appears in PGO-UPy compared to GO, indicating the successful modification of GO nanosheets. The $\mathrm{N} 1 \mathrm{~s}$ spectrum is deconvoluted to three peaks centered at $403.7 \mathrm{eV}$ for $-\mathrm{NH}_{2}$, 
$402.0 \mathrm{eV}$ for $-\mathrm{NH}-$, and $400.4 \mathrm{eV}=\mathrm{N}-$ amine, respectively. The $-\mathrm{NH}_{2}$ and $-\mathrm{NH}-$ peak originates from PDA, while the presence of $=\mathrm{N}$ - groups is assigned to tautomeric species of the intermediates in PDA or UPy groups which serves as a hydrogen acceptor in the formation of hydrogen bonding. ${ }^{1}$

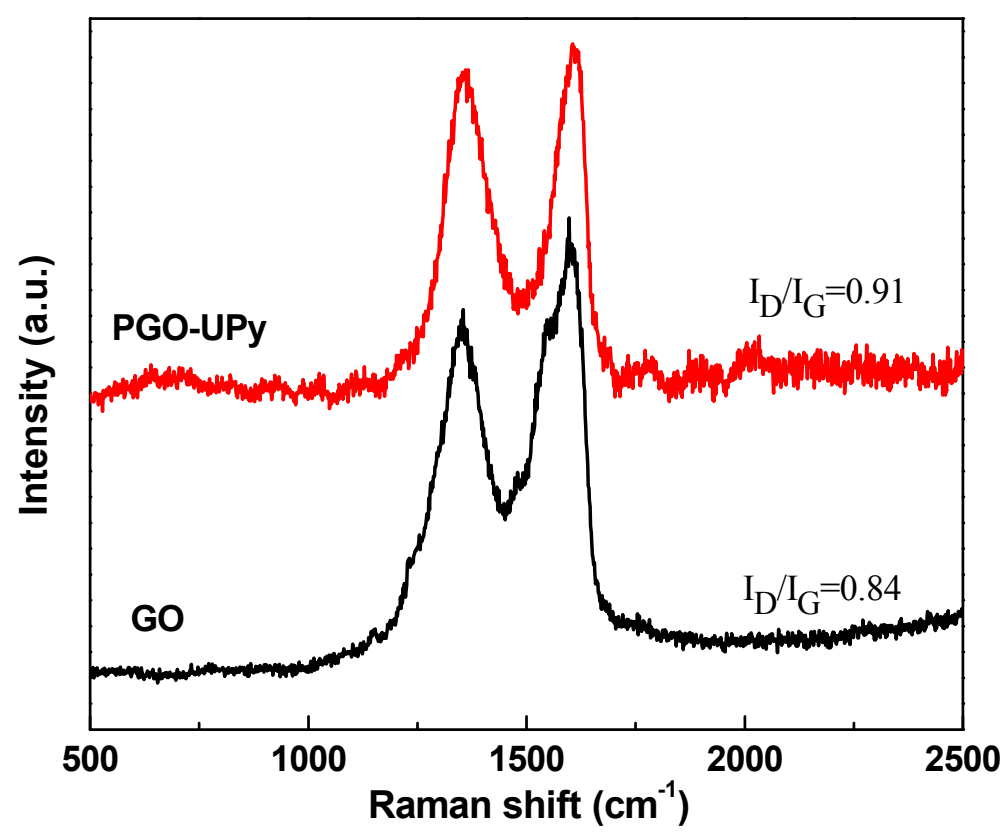

Figure S4. Raman spectra of GO and PGO-UPy.

Figure S4 presents the Raman spectra of GO and PGO-UPy. All samples are of the featured $\mathrm{D}$ and $\mathrm{G}$ bands at $\sim 1351$ and $\sim 1600 \mathrm{~cm}^{-1}$. The $\mathrm{D}$ band is attributed to the carbon $\mathrm{sp}^{3}$ atoms of the defects and disorder structure, and the $\mathrm{G}$ band is related to the ordered carbon $\mathrm{sp}^{2}$ atoms of the graphitic $2 \mathrm{D}$ hexagonal lattice. The value of $I_{\mathrm{D}} / I_{\mathrm{G}}$ could qualitatively characterize the change of defects and disorder structure of graphene-based materials. ${ }^{2}$ The $I_{\mathrm{D}} / I_{\mathrm{G}}$ increases from $0.84(\mathrm{GO})$ to 0.91 (PGO-UPy), confirming the successful functionalization of GO. 


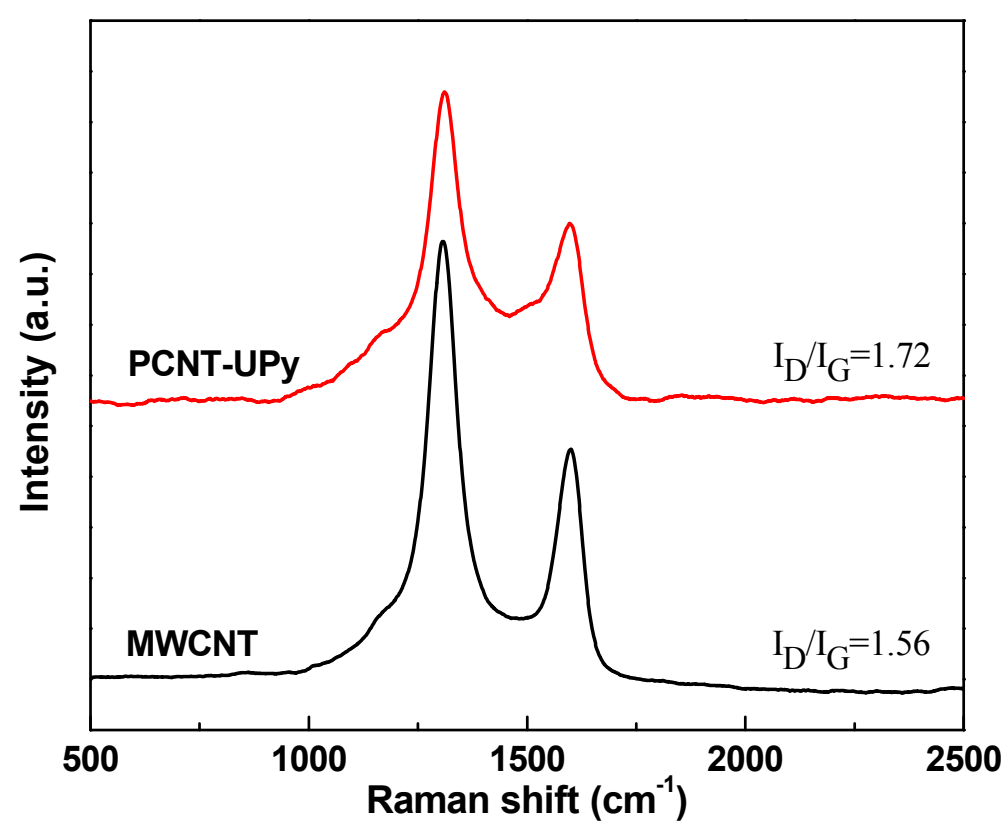

Figure S5. Raman spectra of MWCNT and PCNT-UPy.

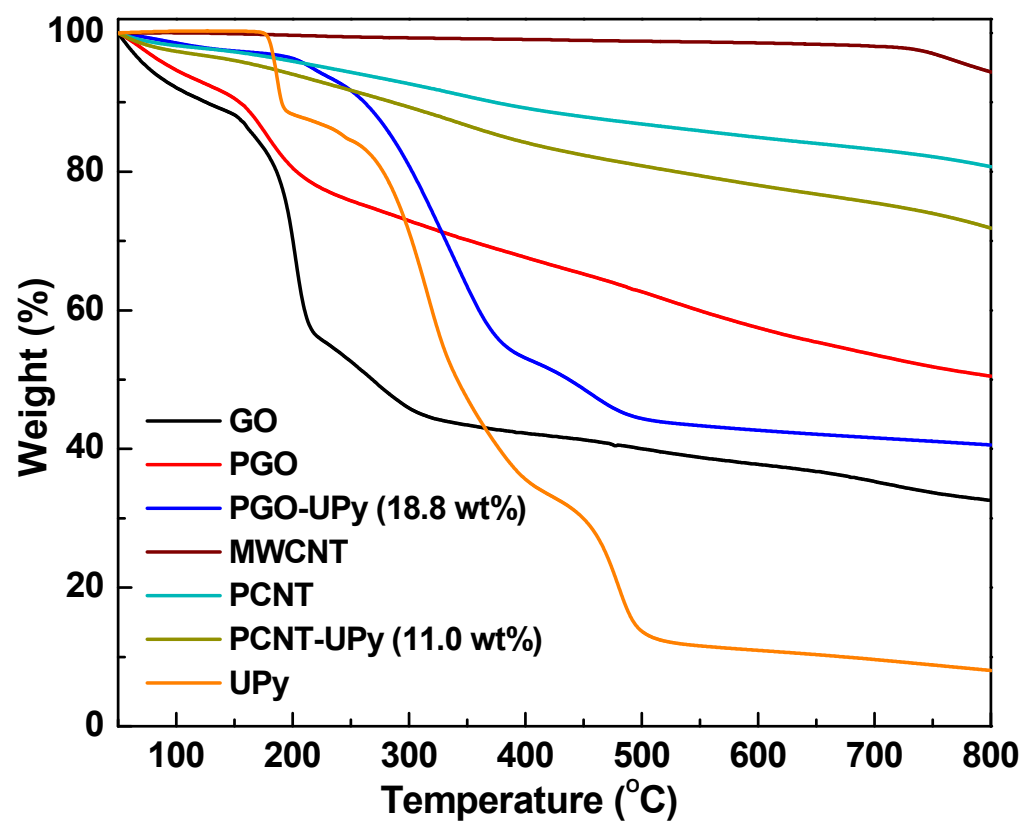

Figure S6. TGA curves of GO, PGO, PGO-UPy, MWCNTs, PCNTs and PCNT-UPy. 


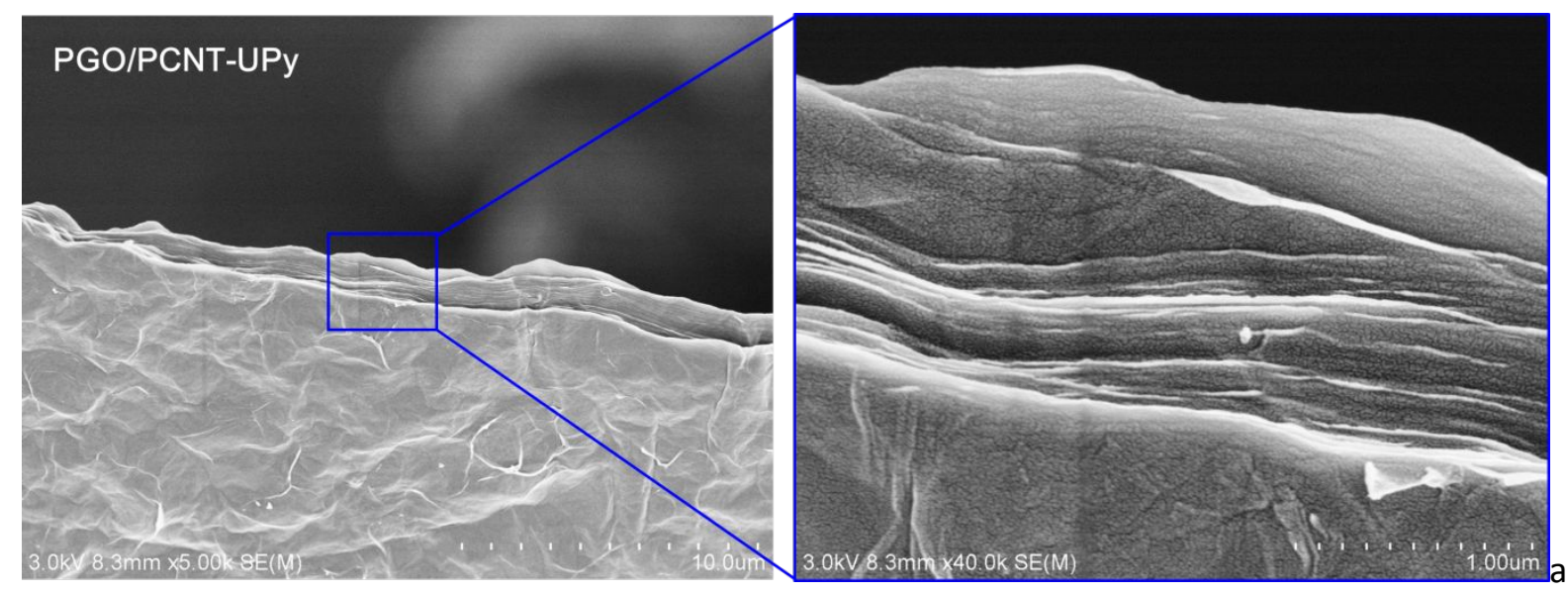

Figure S7. Cross-section SEM images of PGO/PCNT-UPy membrane.

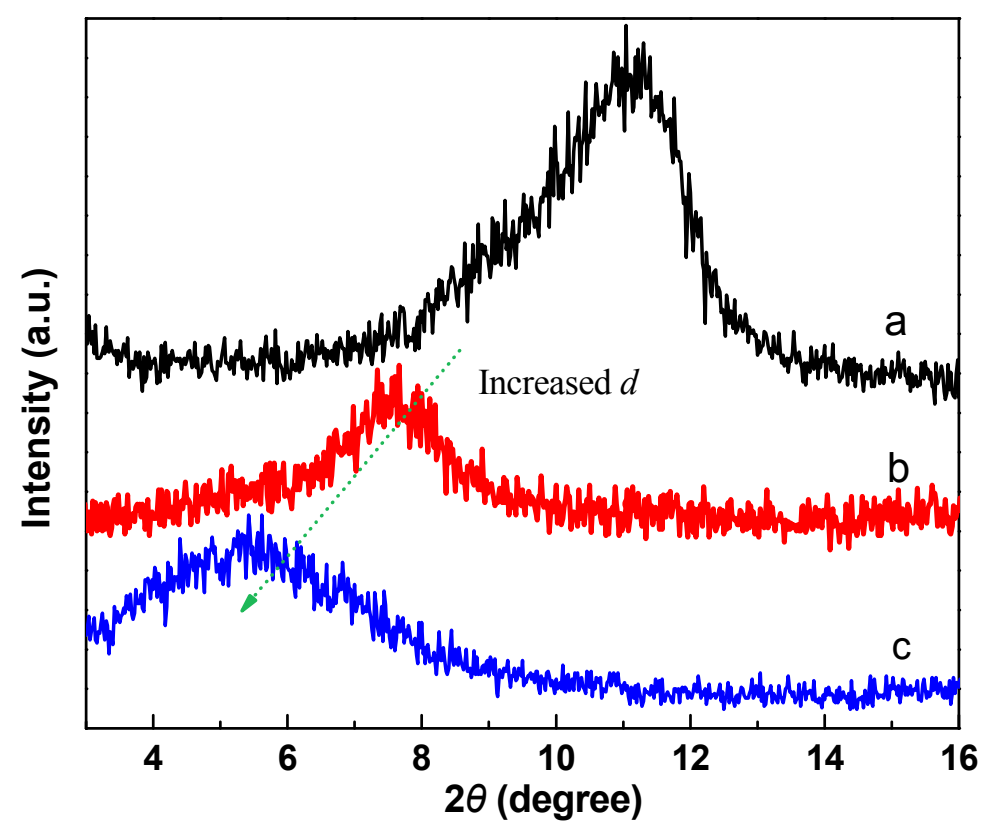

Figure S8. XRD of pure GO membrane (a), and PGO/PCNT-UPy before (b) and after (c) irradiation of NIR.

Figure S8 shows the XRD patterns of pure GO and PGO/PCNT-UPy membrane (the mass ration of PCNT-UPy to PGO-UPy is 1:2) at different states. The interlayer distance ( $d$-spacing) of pure GO film is $\sim 8.09 \AA\left(2 \theta=10.93^{\circ}\right)$, which is well consistent with the previous report. ${ }^{3}$ The PGO/PCNT-UPy membrane was first pre-immersed in deionized water for $30 \mathrm{~min}$. Then this 
membrane was treated with NIR for 30 min for collecting XRD patterns. According to the Bragg equation, the interlayer spacing of PGO/PCNT-UPy increased from $11.83 \AA\left(2 \theta=7.47^{\circ}\right)$ to 16.09 $\AA\left(2 \theta=5.49^{\circ}\right)$ after irradiation of NIR, which is due to the dissociation of quadruple hydrogen bonding.

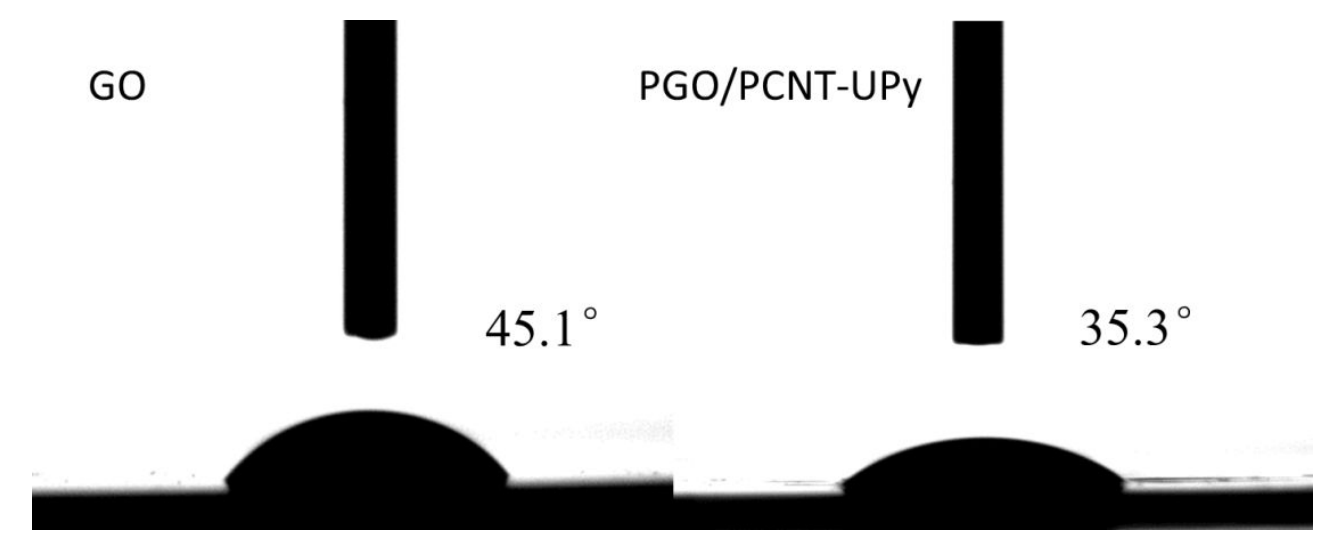

Figure S9. Water contact angle of pure GO and PGO/PCNT-UPy membrane.

As shown in Figure S9, the water contact angle of the pure GO film was measured to approximately $45.1^{\circ}$, the hydrophilicity PDA coating layer can improve the wettability of the membrane surface. The PGO/PCNT-UPy membrane is more hydrophilic with the water contact angles of $35.2^{\circ}$. 


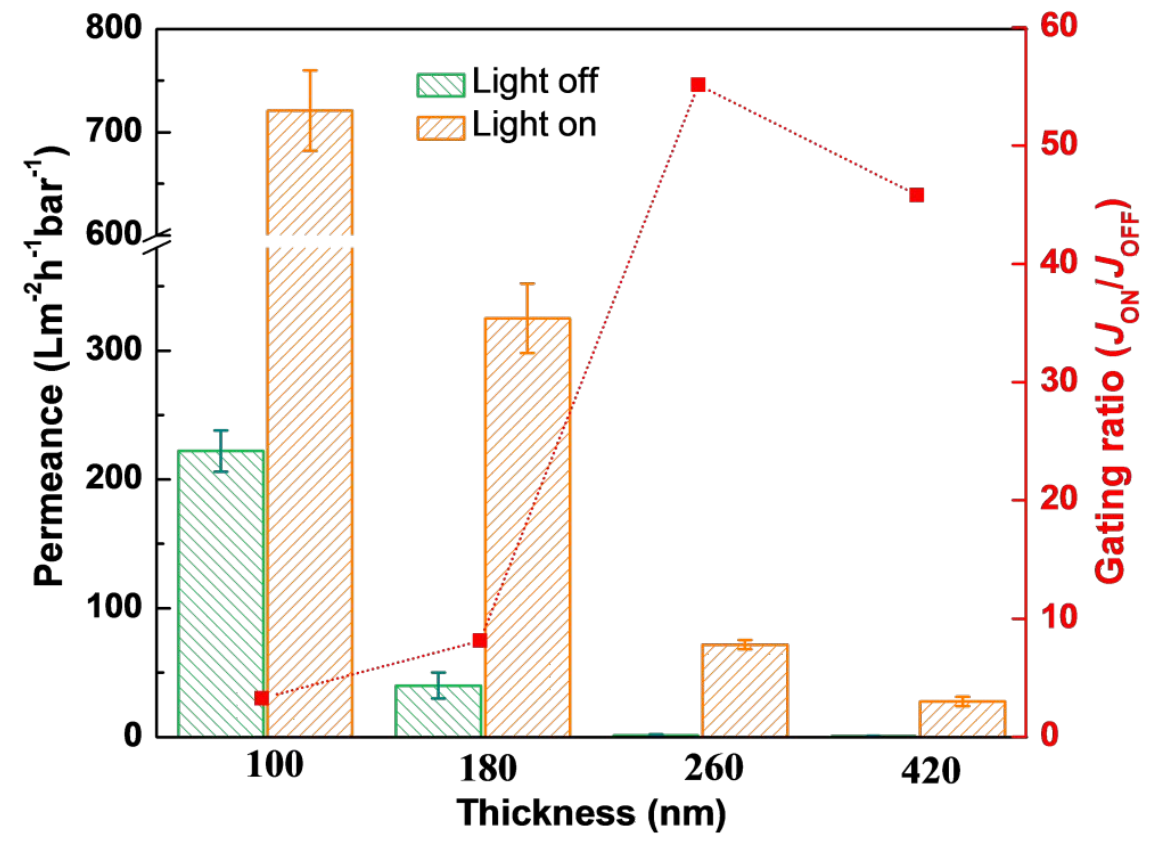

Figure S10. Thickness-dependent water permeance and gating ratio of PGO/PCNT-UPy membranes.

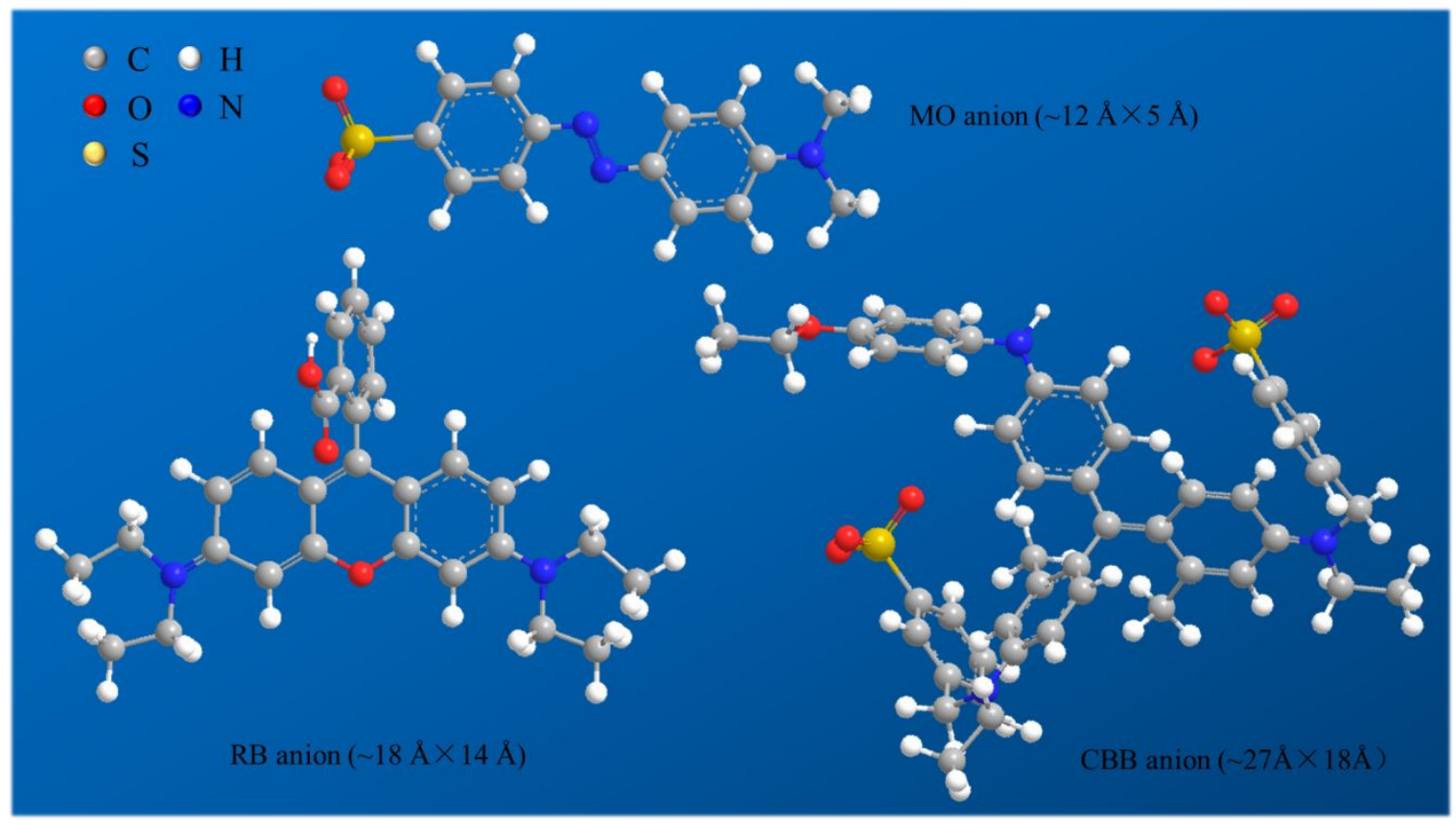

Figure S11. 3D molecular schematic illustrations of methyl orange (MO), rhodamine B (RhB) and coomassie brilliant blue (CBB). 
[1] Zangmeister, R. A.; Morris, T. A.; Tarlov, M. J. Characterization of polydopamine thin films deposited at short times by autoxidation of dopamine. Langmuir 2013, 29, 8619-8628.

[2] Lin, T.; I. Chen, W.; Liu, F.; Yang, C.; Bi, H.; Xu, F.; Huang, F. Nitrogen-doped mesoporous carbon of extraordinary capacitance for electrochemical energy storage. Science 2015, 350, 1508-1513.

[3] Dikin, D. A.; Stankovich, S.; Zimney, E. J.; Piner, R. D.; Dommett, G.; Evmenenko, G.; Nguyen, S. T.; Ruoff R. S. Preparation and characterization of graphene oxide paper. Nature, 2015, 448: 457-460. 